\title{
Research on the Eco-wisdom of Linpan in Chengdu Plain
}

\author{
Tao Jiang \\ College of Landscape Architecture \\ Sichuan Agricultural University \\ Chengdu, China
}

\author{
Xueyang Su \\ College of Landscape Architecture \\ Sichuan Agricultural University \\ Chengdu, China \\ Jing Wang \\ College of Landscape Architecture \\ Sichuan Agricultural University \\ Chengdu, China
}

\author{
Nian Li \\ College of Landscape Architecture \\ Sichuan Agricultural University \\ Chengdu, China \\ Qibing Chen* \\ College of Landscape Architecture \\ Sichuan Agricultural University \\ Chengdu, China
}

\begin{abstract}
The special nature and humanity environment of Chengdu plain gave birth to unique rural settlement 1andscapeLinpan in westen sichuan. The kind of landscape art is a bank of wisdom about the adaptability to the land-use pattern. As regional rural settlement and living art, Linpan is a real reflection of the harmonious relationship between land and its people. It owns much value in landscape aesthetics, landscape ecology, social culture and living art. The article deals with the eco-wisdom of Linpan in the aspects above. The author also analyzed the protection and inheritance of Linpan in modern garden city construction and new rural landscape planning in Chengdu city.
\end{abstract}

Keywords-Linpan; living art; wisdom; Chengdu; rural landscape

\section{INTRODUCTION}

According to the Chinese Academy of Science 2012 China New Urbanization Report, the urbanization of mainland China has reached $51.3 \%$, a substantial increase, exceeding rural population for the first time in history. Industrialized agricultural production methods are replacing traditional farming techniques while the urban community mode of living takes the place of the traditional farming living mode presenting clashes between demand for modern living conditions and preservation of modest physical conditions [1].Contradiction now lies between the expansion of developed areas and the protection of the cropland. Tourism expansion further acts to pressure the rural landscape. Unprecedented challenges urgently face survival of the Chinese rural landscape [2].Chengdu Plain is located in the Dujiangyan irrigation core area with the Minjiang River flowing into the farmland of the plain, forming a highly networked irrigation system.[3]. Located here is Linpan in Chengdu Plain, a unique farming civilization to China and a model of ecological and livable habitation. As a living tradition in Chengdu Plain, Linpan is more and more recognized due to its value in landscape, ecology, art and culture. Development through national urbanization has produced changes for traditional Linpan both in the number of Linpan quantity and Linpan architecture form. Rural residents moving into the town or the rural communities have converted traditional Linpin into an empty nest. In this case, the traditional agricultural civilization is likely in danger of doom. Therefore, study on the ecowisdom of Linpan is of important theoretical and practical significance under the background of urbanization in Chengdu.

\section{FIELD STUDY OF ECO-WISDOM IN LINPAN}

The unique village landscape pattern of Linpan is developed in the specific natural and cultural environment in Chengdu plain. It reveals the adaptability relationship between western Sichuan residents and the natural processes and patterns impacting the western Sichuan plain during the process of living and production. Linpan is not only a model for sustainability but also crystallizes western Sichuan farming civilization, reflecting harmonious relations between man and the land.

This paper presents the argument that harmony achieved between residents and nature in Linpan is through residential compliance with nature; hence, "eco-wisdom" in Linpan, arises. eco-wisdom is studied to answer the following questions: What contributes to Linpan's long-term existence? How do local farmers exist in harmony with nature and survive? What survival wisdom do they convey? How can society put

*Corresponding author. Qibing Chen 
this wisdom to use to assure the survival of Linpan in a rapid urbanization? "Fig. 1"

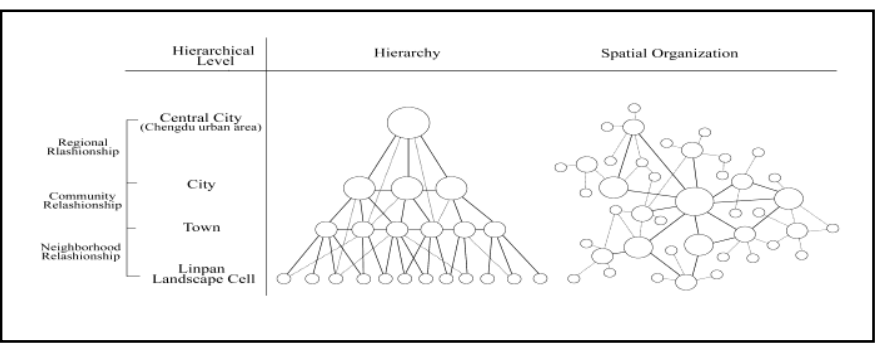

Fig. 1. An aerial view of Linpan landscape units

The study of western Sichuan Linpan eco-wisdom can expand from both macro and micro levels. On the macro level, the causes and effects elements can be considered from morphology, while on micro, the survival wisdom in the production and the living can be concluded and analyzed in terms of the farmers.

The field study is conducted from two aspects: the ecowisdom in production and the eco-wisdom in living. In each aspect the wisdom of "Lin, Shui, Zhai, Tian" (they are the landscape elements of Linpan) is explored. The objects of the study of eco-wisdom in production include the forest, agricultural irrigation, agricultural buildings, farmland and crops, and the production technology; as for in living, it includes: Fengshui forest, water and sewage, residential buildings and other buildings, road systems, etc.

\section{ECO-WISDOM OF LINPAN IN MACRO VSPATIAL MORPHOLOGY}

The spatial morphology of Linpan falls into two levels: the Linpan settlement and the Linpan landscape units. From the viewpoint of Linpan settlements, Chengdu Plain urban spatial structure system can be divided into 4 levels, namely, the city center (Urban District, Chengdu), the city (Chengdu city districts and counties), the town (township level), and the Linpan (village level). "Fig. 1" The system of Linpan settlement is composed of the four levels above; the space combination pattern has strong self similarity and scale free characteristics. The Linpan settlement landscape is formed and developed in the long-term interaction of natural and social factors, and its morphology is characteristic "Fig. 2", namely, the slug decentralized layout mode [4].

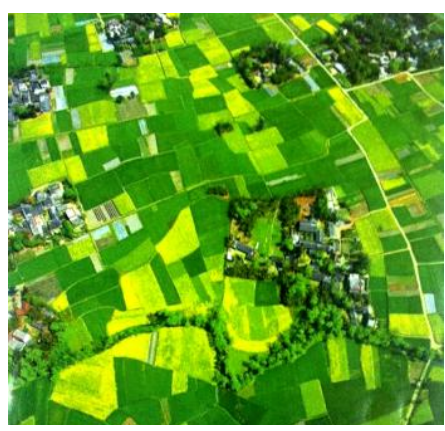

Fig. 2. City and countryside spaces framework of Chengdu plain.
From the perspective of the forest landscape units, Linpan is composed of the gardens, the housing and the periphery of the cultivated land. Its elements are Lin (grove), Shui (water system), Zhai (courtyard \& residences), and Tian (farmland) "Fig. 3". From the level of the spatial form of Linpan, its spatial structure includes the field, the forest, the house, and corresponds to the architecturally virtual space, the gray space and the real space, respectively. From the vertical space form of Linpan, its spatial structures are the tree canopy layer, the house, and the paddy fields and the surface layer. The combination of the 3 levels improves use efficiency of the energy and the material: the upper layer absorbs solar energy to regulate the climate, the middle provides venues, and the lower ensures energy supply.

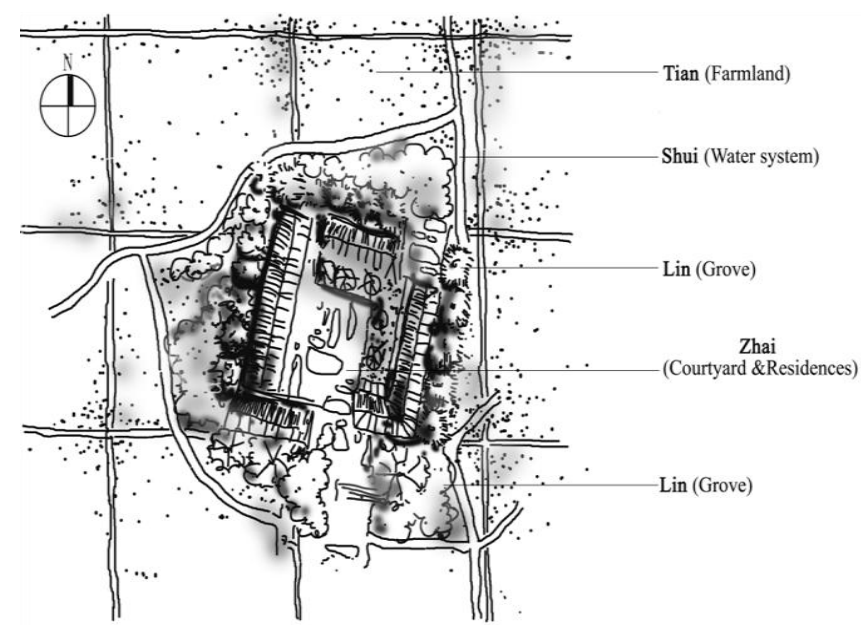

Fig. 3. Floorplan of Linpan landscape units.

\section{ECO-W ISDOM OF LINPAN IN MICRO WESTERN SiCHUAN PEOPLE 'S SURVIVAL WISDOM}

\section{A. The Survival Wisdom in Production}

- Zhai: From the horizontal space, while the size of the forms are different, the internal space has reasonable division and arrangement to meet the needs of the living, the cultivation, the storage, the processing, and other production functions. From the layout of the house, the Linpan construction mainly includes "one" shaped courtyards, "L" shaped courtyards, threesection compound or four-section compound form "Fig. 4". The work space (such as agricultural produce processing) is according to the form of Linpan's layout, and it is separated from the living space with convenience accounted for. Wang $\mathrm{Si}$ town $\mathrm{Xi}$ 'an village in Dayi for example, home storage space (storing farm tools, etc.) is located near the entrance, allowing clear separation between the yard and the living space, and adding convenience for the cultivator to use the production tools. Drying racks made of local bamboo are efficiently stored under the eaves of exterior walls. 
- Shui: The Dujiangyan irrigation project allows Linpan to have many water networks and a small wetland system, which not only provides the habitat for small animals and plants, but also meets the farming requirements of people. The Chengdu plain has many water networks. The main streams and tributaries, the lateral canal, and the sublateral network jointly build the drainage system. Around the Linpan settlements there are a large number of different sizes of pond which are used to collect rain water and underground water to irrigate the farmland, an important role in improving Linpan biodiversity and the microclimate [5].

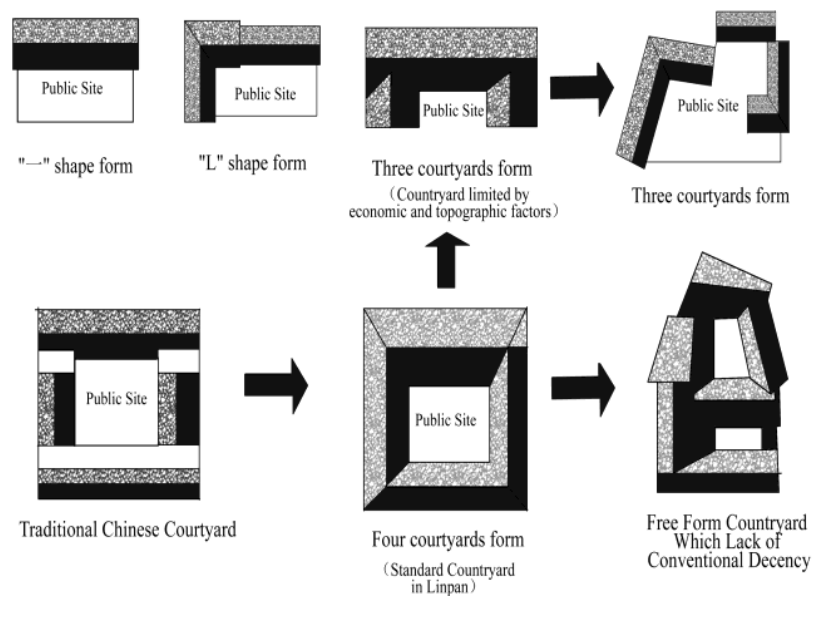

Fig. 4. Several kinds of compound forms of Linpan in Chengdu Plain

- Lin: Bamboo is commonly found in the forest. Linpan incorporates bamboo into buildings reflecting an aesthetic taste of the natural landscape garden. Bamboo forests not only improve the local microclimate by acting to maintain the carbon and oxygen balance to clean the air, but they also act as an important economic source of income for the local residents. For example, many villagers in Guangxing town pagoda village in Jintang make bamboo weaving crafts, and engage in bamboo breeding.

- Tian: Linpan's constructed wetland system, conforms to the landscape ecology of the "patch, corridor, matrix" theory. The settlement, the forest land, and the pond are patches. The road, the forest, and the irrigation system are corridors and the farmland is the general matrix. In addition, the junctions of the farmland and the woodland are in accordance with the "edge effect" of some characteristics, and play an important role in the biodiversity and the improvement of the ecological environment. They could provide food and shelter for wildlife. The distance from the farmland to the Linpan building is within $300 \mathrm{~m}$, which is suitable for walking without feeling tired, and also convenient for Linpan residents to manage the farmland [6].

\section{B. The Survival Wisdom in Life}

1) Zhai: Linpan buildings are often selected consistently with the pattern of FengShui in the location of "surface water back mountain" which is ideal landscape for Chinese people. Linpan construction combines different materials to create rich layout forms reflective of the philosophy "no violation of nature and in use of nature"[7]. For example, there are wending canals in Pixian Anlong village Linpan, and tall trees and bamboo behind the house. Linpan buildings are made of the rich local wood, the bamboo, the grass, the stone and other natural materials, as well as, grey tile, brick and other artificial materials. This is economical and creates a natural and simple aroma. Linpan architectural interior spaces are built rationally around the small yard which can be used to dry and thresh crops, constitutes a space full of affinity, compact and pleasantness.

2) Shui: The water system which originated from Dujiangyan irrigation and the road system of Linpan in Chengdu Plain settlement system is complementary to each other, emulating a phenomenon of water going after the road and the road going after the water, to form a rich water space environment. For example, many Linpan located are around ponds in the Jintang County Guang Xing Zhen Baota village. The canal in Xingjing county Xingyi town Sanhe village is next to Linpan buildings. The affinity for water makes the pond a preferred location for parks and recreation. People gather near the water to engage in a variety of social activities; all of these imply a close relationship between the water and the Linpan.

3) Lin: The evergreen broad-leaved forest and bamboo are among main vegetation types of Linpan, which not only play an important ecological role, but also maintain landscape and cultural value. There is no clear path under forest, and it is formed by the villagers for convenience, and it is complex and fuzzy for the specific use function..For example, there is a large bamboo forest in Xiangyang village in Qionglai City, we can carry out various activities such as drinking tea, having a rest and taking exercises and so on, and the space becomes "Lin FengShui", an ideal place for the villagers, and gives people a sense of belonging.

4) Tian: Farmland has a special aesthetic value at the base of Linpan. Farmlands often symbolize farming culture of the West Sichuan pastoral scenery. Terrace beauty is the vast land art of agricultural landscape, bears the farming culture of the West Sichuan pastoral scenery. Linpan in autumn and winter is dry land farmland, and in spring and summer is the paddy field. The farmland crops are rich in types, always changing color and providing visual stimuli.

\section{ACKNOWLEDGMENT}

This paper is a partial product of the research project funded by the National Natural Science Foundation of China subsidization project. The project is study on the coupling mechanism of the human physiological and psychological response to the ornamental bamboo and its health function 
(project code 31570700). This paper is also funded by the Key techniques research and demonstration of Sichuan rare ornamental bamboos cultivation (project code 2012220037) of industry-university-research innovation alliance and the Research and Teaching Enhancement Grant from school of landscape architecture, Sichuan agricultural university.

\section{REFERENCES}

[1] Antrop, M, “ Landscape change and the urbanization process in Europe.,"Landscape and Urban Planning,vol.67,pp.9-26,March 2004.

[2] Kristensen S , “Multivariate analysis of landscape changes and farm characteristics in a study area in central Jutland, ”Ecological Modelling, vol.168,pp.303-318,October 2003

[3] Fang Z.R., Li X.K, "The historical origin of Linpan culture in western Sichuan,"Journal of Chengdu University (SOCIAL SCIENCE EDITION), vol.5,pp. 45-49, October 2011.

[4] Duan, P., Liu, T.H., Ecological homland of Shu culture.Sichuan Science and Technology Press, 2004.

[5] Chen,Q.B., Research on landscape resources protection and development mode of Linpan in Chengdu Plain. China Forestry Press, 2011.

[6] Zheng, J., "Study on the ecological significance of Linpan in western Sichuan province,"Shanxi architecture,vol.36,pp.50-53,December 2010.

[7] Hong Sun-Kee, Song In-Ju, Wu, J.G., "Fengshui theory in urban landscape planning,”Urban Ecosyst,vol.10,pp.221-237,October, 2007. 